International Journal of Robotic Computing

Vol. 3, No. 1 (2021) 1-21

(C) KS Press, Institute for Semantic Computing Foundation

DOI: $10.35708 / \mathrm{RC} 1870-126263$

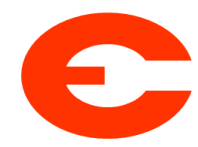

\title{
Packing Planning and Execution Considering Arrangement Rules
}

\author{
Akiya Yasuda, Gustavo Alfonso Garcia Ricardez, Jun Takamatsu, and Tsukasa \\ Ogasawara \\ Nara Institute of Science and Technology at 8916-5 Takayama, Ikoma, Nara \\ 630-0192, Japan \\ \{yasuda.akiya.xs2, garcia-g,j-taka, ogasawar\}@is.naist.jp
}

Received (03/15/2021)

Accepted (03/31/2021)

\begin{abstract}
To send a consumer the ordered products in e-commerce, it is necessary to pack the products into a container. When packing, we need to consider the arrangement of the products to avoid damaging them during transportation by using the know-how of packing. In this paper, we propose a packing robot system that plans the arrangement and then executes it. To develop this robot system, we employ two ideas. The first idea is to split the whole process into the arrangement of the objects and robot motion generation. The arrangement is regarded as a $3 \mathrm{D}$ bin-packing problem. The second idea is to use the heuristics of the packing so that objects are stacked from the bottom. Since the space above the target position is free from collisions, the strategy to approach from the top reduces the possibility of a collision. In the experiments, we succeeded in arranging eleven objects satisfying the assigned rules and packing ten of the eleven objects using the robot.
\end{abstract}

Keywords: Bin packing; manipulation; box loading.

\section{Introduction}

As e-commerce trade increases, one would like robots to automate the tasks for warehouse and retail operations. For example, to send a consumer the ordered products, it is necessary to pack the products into a container.

To develop packing robots, there are two difficulties: one difficulty is that robots need to manage many types of products, since preferences of individuals are diverse. The other difficulty comes from the requirement to avoid damaging the products during transportation. Therefore, we need to arrange the products following the know-how of packing. 


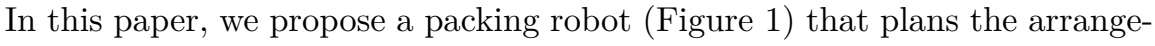
ment and then executes it. The packing is similar to the Pick task in the Amazon Robotics Challenge (ARC) [I]. In our target task, the ratio between the volume of the sum of all the items and that of the container is larger. This forces to put some objects on top of other objects. First, we input the ordered objects, their physical properties (e.g., dimension, mass, type of rigidity), the size of the container, and the know-how that is represented as if-then rules. The method calculates an arrangement that satisfies the rules and then generates the robot motion to achieve the arrangement. We assume that the shape of each object can be represented by approximating it to a rectangular solid or cubiod, that the way to grasp the object is known a priori, and that the order of packing is given. To decide the order automatically is future work.

To achieve a packing robot, we employ two ideas. The first idea is to split the whole process into the arrangement of the objects and robot motion generation.

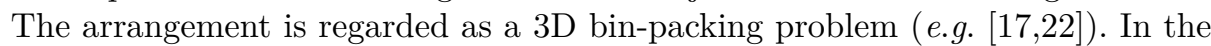
motion generation, the DOF of the planning can be reduced by assuming the already placed objects as a static environment. The second idea is to use the heuristics of the packing so that objects are stacked from the bottom. Since the space above the target position is free from collisions, the strategy to approach from the top reduces the possibility of collisions.

\section{Related Works}

Pick-and-place tasks are still challenging in robotics [118], as organized in recent robot competitions such as the ARC [ח, $4,2,[,[1,5,5]$. In the ARC, robots must pick multiple items from a cluttered container and orderly place them in a shipping box without letting any item protrude out of the box neither crushing the previously placed items. Though the placing is similar to our target task, the task in ARC is achieved by just searching a vacant space and placing.

In the arrangement, King et al. [II] proposed object-centric and robot-centric actions; the former is the action executed on the object, and the latter is the actions related to the robot itself such as moving closer or farther from the objects. Nebel et al. [20] proposed a household robot that handles the uncertainties of an environment by repeating sensing a part of the environment and replanning the actions.

The packing, arrangement of the objects in the predefined space, is regarded as a 3D bin-packing problem [ए]]. In most cases, the problem is solved under the assumption that the shapes of the objects are rectangular solids and the dimensions are given. The problem is classified based on the variety of the objects and whether the size of the space is chosen or not [22].

Li et al. [153] proposed to formulate the packing problem as a kind of optimization. The proposed method extends this method to adopt the rules for arrangement and to realize stable arrangement. Iwasawa et al. $[8]$ proposed the representation of arrangement Sequence-Triple. This representation efficiently represents a valid arrangement.

After planning the arrangement, we use motion planning such as [9, [2] to generate robot motions to achieve the arrangement. Thus, the proposed method 
is a kind of symbolic-and-motion planning [ [ $]$ ] and knowledge processing [ [ZI]. The proposed method targets the robot packing. To alleviate the difficulty, the proposed method well considers the characteristics of packing tasks.

To plan the grasp only from the object shape is out of scope of this paper. In recent progress of deep learning technologies, Mahler et al. [14,,I5] have proposed a deep neural network to plan the grasp.

\section{Packing Arrangement}

\subsection{Overview}

Given the information of objects to be packed and the container, the task of the packing arrangement is to decide the configurations of all the objects. We regard the arrangement as a 3D bin-packing problem and employ the method [1.3] that decides the configuration by maximizing the objective function. The objective function positively evaluates the preferable configurations and heavily penalizes the cases where the rules are broken.

Given the positions of all the objects $\mathbf{p}_{i}=\left(x_{i}, y_{i}, z_{i}\right)$, the objective function $F$ is defined as Equation (四).

$$
F=\omega_{1} F_{\text {overlap }}+\omega_{2} F_{\mathrm{COG}}+\omega_{3} F_{\text {contact }}+\omega_{4} F_{\text {type }} .
$$

Each function in the right-hand side evaluates the preference of arrangement or penalizes the arrangement and $\omega_{*}$ weighs each function.

The functions $F_{\text {overlap }}$ and $F_{\mathrm{COG}}$ were defined in [13]. The former penalizes the penetration of objects and the latter controls the center-of-gravity (COG) position. We define the new functions $F_{\text {contact }}$ and $F_{\text {type }}$. The former aims to realize stable arrangement of objects and the latter penalizes the cases when the rules of the arrangement are violated.

We assume that the object is oriented with its axes aligned to the axes of the container. Thus, the orientation of an object is one of six patterns. Since it is difficult to estimate the positions and orientations simultaneously, we optimize the positions for all the combinations of the orientations.

Like in the previous method [13], we use the particle swarm optimization (PSO) [IU] to maximize the objective function. Since the penalty function abruptly changes its value, the optimization using the gradient of the function is not suitable. The PSO optimizes the function without using gradients. The PSO distributes particles and each particle searches for the optimum by cooperating with the others.

\subsection{Preliminaries}

The information of an object consists of the dimension $\left(W_{i}, D_{i}, H_{i}\right)$, the mass $m_{i}$ and the type that is related to the rule. We would also like to define the overlapping length along the $x$-, $y$-, and $z$-axes. Consider the $x$-axis case. Let the minimum and the maximum value of the $x$-position of the object $i$ be $x_{\min , i}$ and $x_{\max , i}$. For the pair of objects $i$ and $j$, the overlapping length is the length of 
the intersection between $\left[x_{\min , i}, x_{\max , i}\right]$ and $\left[x_{\min , j}, x_{\max , j}\right]$. Using the positions and the dimensions, the overlapping length $o l_{i, j}^{x}$ is defined as Equation (ㅁ).

$$
o l_{i, j}^{x}= \begin{cases}0 & \left(d_{i, j}^{x} \geq\left(W_{i}+W_{j}\right) / 2\right) \\ \min \left\{W_{i}, W_{j}\right\} & \left(d_{i, j}^{x}<\left|W_{i}-W_{j}\right| / 2\right) \\ \left(W_{i}+W_{j}\right) / 2-d_{i, j}^{x} & \text { (otherwise) }\end{cases}
$$

where $d_{i, j}^{x}=\left|x_{i}-x_{j}\right|$. Similarly, we can define the length along the $y$ - and $z$-axes: $o l_{i, j}^{y}, o l_{i, j}^{z}$.

\subsection{Maximizing Supporting Areas}

A stacking object is more stable as the supported area becomes larger, i.e., the unsupported area $s_{i}$ becomes smaller. There are three cases: 1) the object $i$ is placed onto the object $j, 2$ ) the object $i$ is placed onto the bottom of the container, i.e., $z_{i}=H_{i} / 2$, and 3 ) there are no objects in contact. For the three cases, $s_{i}$ is defined as Equation (3).

$$
s_{i}= \begin{cases}\left(W_{i} \times D_{i}\right)-\left(o l_{i, j}^{x} \times o l_{i, j}^{y}\right) & (\text { Case } 1) \\ 0 & (\text { Case 2) } \\ W_{i} \times D_{i} & (\text { Case 3) }\end{cases}
$$

We define the function $F_{\text {contact }}$ as Equation (四).

$$
F_{\text {contact }}=\frac{1}{S_{\text {contact }}+\epsilon_{\text {contact }}},
$$

where $S_{\text {contact }}=\sum_{i=1}^{N} s_{i}, N$ is the number of objects, and $\epsilon_{\text {contact }}$ is a predefined small value to avoid zero-division.

\subsection{Rules for Arrangement}

Given the algorithm to determine if the object $i$ violates the rule $k$ for the arrangement, the variable $c_{k i}$ is defined as follows:

$$
c_{k i}= \begin{cases}a_{k} & \text { (the rule is violated) } \\ 0 & \text { (otherwise) }\end{cases}
$$

where $a_{k}$ is the degree of the penalty. Similarly to the other functions, we define the function $F_{\text {type }}$ as Equation ([).

$$
F_{\text {type }}=\frac{1}{C+\epsilon_{\text {type }}},
$$

where $C=\sum_{k=1}^{K} \sum_{i=1}^{N} c_{k i}, K$ is the number of rules, and $\epsilon_{\text {type }}$ is a predefined small value. 


\subsection{Other Functions}

The remiaining two functions are defined in [133]. The first function penalizes that one object penetrates another object. The penalty of the penetration for objects $i$ and $j$ is defined using the overlapped volume as

$$
v_{i, j}=o l_{i, j}^{x} \times o l_{i, j}^{y} \times o l_{i, j}^{z} .
$$

The function $F_{\text {overlap }}$ is defined as

$$
F_{\text {overlap }}=\frac{1}{V_{\text {overlap }}+\epsilon_{\text {overlap }}},
$$

where $V_{\text {overlap }}=\sum_{i=1}^{N-1} \sum_{j=i+1}^{N} v_{i, j}$ and $\epsilon_{\text {overlap }}$ is a predefined small value.

The second function attempts to arrange the objects such that the COG is located near the center of the bottom $\mathbf{C}$ of the container. The COG of all the objects is defined as

$$
\mathbf{G}=\left(\frac{\sum_{i=1}^{N} x_{i} m_{i}}{\sum_{i=1}^{N} m_{i}}, \frac{\sum_{i=1}^{N} y_{i} m_{i}}{\sum_{i=1}^{N} m_{i}}, \frac{\sum_{i=1}^{N} z_{i} m_{i}}{\sum_{i=1}^{N} m_{i}}\right) .
$$

The function $F_{\mathrm{COG}}$ is defined as

$$
F_{\mathrm{COG}}=\frac{1}{d_{\mathrm{COG}}+\epsilon_{\mathrm{COG}}},
$$

where $d_{\mathrm{COG}}=\|\mathbf{G}-\mathbf{C}\|$ and $\epsilon_{\mathrm{COG}}$ is a predefined small value.

\section{Generating Packing Motions}

\subsection{Motions of Manipulator}

To succeed in executing the planned arrangement as is, we employ two heuristics. First, we put objects from the bottom. Since the space above an object tends to be free from obstacles, we approach the target position from above. Second, to reduce the possibility to collide with other objects, the manipulator attempts to grasp an object from above. If an object is difficult to grasp from above, we pack it earlier before filling the space with other objects. As described in Section $\mathbb{W}$, the order of packing is manually assigned following the heuristics.

In each step, the initial position and the target position (from the planning) of the object, and the way of grasping (e.g., the relative position between the object and the end effector) are given. First, we set an intermediate position of the object to above of the target position. Next, we calculate the configurations of the end effector at the initial, intermediate, and the target positions. Then, we calculate the configurations of the manipulator at the three positions using inverse kinematics. Finally, we generate the trajectory from the initial to the intermediate positions using common path planning [112] and that from the intermediate to target positions by moving straight to the object.

The aforementioned motion generation provides two advantages. First, relying on the heuristics about the above space, the straight path from intermediate to target positions tends to be free from collision. Second, we accelerate the motion generation by skipping the complicated path planning in the complicated arrangement around the target position. 


\subsection{Redundancy of Grasping}

In this paper, a suction gripper is used. Figure $\square$ shows the example of grasping an object. The axis of the end effector should be aligned along the normal direction and we have one DOF around the normal, i.e., $\theta_{z}$. We discretize the angles and generate several candidates of the motions to find a collision-free path by changing the grasp, i.e., $\theta_{z}$.

\section{Robot System}

\subsection{Hardware}

Figure $\mathbb{U}$ shows the robot hardware that we used in our experiments. The hardware consists of a robot arm and an end effector (see also Figure [1]). The arm

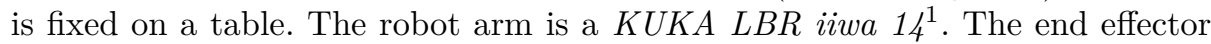
is custom designed in cooperation with Panasonic Corporation [6]. The end effector consists of a suction cup to grasp an object, a joint motor to control the direction of the cup, a linear passive joint to absorb the impact of contact, and an RGB-D camera Intel RealSense D415.

\subsection{Software}

We implement the system on the Robot Operating System ${ }^{\square}$ (ROS). We use Ubuntu 16.04 as the operating system and the version of ROS is Kinetic. We use the library librealsense to capture RGB and depth images from the RealSense camera. To ease object detection and localization, we use AR markers through the ROS package ar_track_alvar. For the simulation experiments, we use Gazebo 9.0

For motion planning, we use RRT-Connect [1]2] implemented on the motion planning framework MoveIt! [3]. We check the reachability of the robot arm using the reachability map [[6]. Thanks to this map, we quickly determine if the arm cannot reach the target position due to its kinematic limitation and thus accelerate the whole process of motion planning.

\section{Experiments}

\subsection{Setup}

We define three types of objects as Rigid, Deformable, and Breakable. Type Rigid represents objects that are hard to penetrate or deform. If a rigid object falls onto another object, the rigid object possibly breaks the other object. Type Deformable represents objects that can easily bend, twist or that are easy to penetrate. Any objects that are placed onto the deformable object are unstable. Type Breakable represents objects that are fragile. Some objects have more than one type, such as deformable and breakable.

Considering the characteristics of the object types, we assign the following three rules:

\footnotetext{
${ }^{1}$ https://www.kuka.com/en-us/products/robotics-systems/industrial-robots/lbriiwa

2 http://www.ros.org/

${ }^{3}$ http://gazebosim.org
} 
Table 1: Constant values

\begin{tabular}{|c||c|c|c|c|}
\hline Parameter & $\epsilon_{\text {overlap }}$ & $\epsilon_{\mathrm{COG}}$ & $\epsilon_{\text {contact }}$ & $\epsilon_{\text {type }}$ \\
\hline Value & 0.000001 & 0.001 & 0.001 & 1 \\
\hline
\end{tabular}

Table 2: Weights of optimized function

\begin{tabular}{|c||c|c|c|c|}
\hline Parameter & $\omega_{1}$ & $\omega_{2}$ & $\omega_{3}$ & $\omega_{4}$ \\
\hline Value & 10000000 & 800000 & 500000 & 300000 \\
\hline
\end{tabular}

1. Do not place any objects under a rigid object.

2. Do not place any objects onto a deformable object.

3. Do not place any rigid objects around a breakable object.

In each rule, we set the penalties as $a_{1}=3, a_{2}=1, a_{3}=3$. We set the predefined small value $\epsilon$ and the weight $\omega$ of the objective function as shown in Tables $\square$ and 四.

\subsection{Target Items}

We use a container whose dimension is $320 \times 270 \times 360[\mathrm{~mm}]$. Tables $\mathbf{B}$ to $\mathbf{b}$ show the eleven objects used in the experiments. We regard Snack $B$ as the breakable and deformable object, since the package is easily deformed depending on its orientation. The objects which do not belong to the three types are referred as others and shown in Table [. We chose these items from the objects used in the ARC 2017.

\subsection{Real-robot Experiments}

Resulting Arrangement. We compare the proposed method to the method [13]. The difference is that the compared method does not use the functions $F_{\text {contact }}$ and $F_{\text {type }}$. In both methods, we reject the results where the overlapped volume $V_{\text {overlap }}$ is not zero, i.e., one object penetrates another object.

Figures $⿴$ and 1 show the result of packing arrangement with the compared and proposed methods, respectively. Colors represent the object identities (see Tables [1] to to know the correspondences). Figure (c) shows the process of packing and helps to understand the arrangement of the proposed method. The proposed method arranges the rigid objects to the bottom and makes the area to support snacks using the rigid objects and the other objects. Finally the proposed method put the snacks in the higher space. On the other hand, the compared method does not consider the arrangement of deformable objects (green rectangles).

Table $\mathbb{Z}$ shows the quantitative comparison of the arrangement. Since the unsupported area of the object in the proposed method is smaller than that in the compared method, we conclude that the arrangement of the proposed method is more stable. Though the proposed method follows the rules in the arrangement, the compared method violates the rules twice. We found that the height of COG in the proposed method is slightly higher than that in the compared method $(0.138[\mathrm{~m}]$ to $0.114[\mathrm{~m}])$. 
Table 3: Rigid objects

\begin{tabular}{|c|c|c|}
\hline Name & Canned food & Coffee bottle \\
\hline Picture & & \\
& & \\
& & \\
& & \\
\hline Weight $[\mathrm{g}]$ & 681 & 570 \\
\hline Size $[\mathrm{mm}]$ & $77 \times 77 \times 196$ & $90 \times 90 \times 175$ \\
\hline Color & & \\
\hline
\end{tabular}

Table 4: Deformable objects

\begin{tabular}{|c|c|c|}
\hline Name & Liquid detergent & Powder pouch \\
\hline & & \\
& & \\
& & \\
& & \\
& & \\
& & \\
\hline Weight $[\mathrm{g}]$ & & \\
\hline Size $[\mathrm{mm}]$ & $155 \times 80 \times 235$ & $140 \times 70 \times 200$ \\
\hline Color & & \\
\hline
\end{tabular}

Resulting Robot Execution. We put the target objects whose upright direction is the same in the final configuration on a working table one by one. The robot picks up and places the object into the container repeatedly. We evaluate the proposed method in the simulation and in the actual environment.

In the simulation, we verify if the generated motion is valid, i.e., the motion is free from collisions. Though we applied only one result obtained by the proposed arrangement method, the simulator verified that the generated motion succeeded in the execution without any collisions.

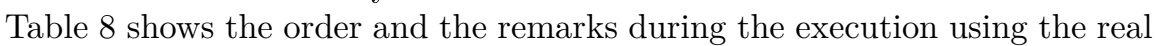
robot. Figure [ shows the motion to put the Speed Stick and the process of packing. Please watch our video ${ }^{\mathbb{T}}$, which shows the whole execution. The task is successfully executed except for packing the tissue box. Due to the errors on

\footnotetext{
${ }^{4}$ https://www $\cdot$ youtube.com/playlist?list=PLdyaJdngypBip8r606PF4bxH_ IOZIPIIN
} 
Table 5: Breakable objects

\begin{tabular}{|c|c|c|}
\hline Name & Snack A & Snack B \\
\hline & & \\
Picture & & \\
& & \\
& & \\
& & \\
\hline Weight $[\mathrm{g}]$ & 181 & 138 \\
\hline Size $[\mathrm{mm}]$ & $150 \times 240 \times 42$ & $170 \times 52 \times 250$ \\
\hline Color & & \\
\hline
\end{tabular}

vision and the differences between the simulation and the actual environment, 1) the canned food and coffee bottle are placed in a slightly different position horizontally, 2) the tissue box is colliding with a stacked object, and 3) snack $\mathrm{A}$ is released slightly above the target position. We confirmed that the robot executed the curved motion in spite that the straight motion is generated in the simulation. It is a future work to fill the gap between the simulation and the actual environment.

\subsection{Simulation Experiments}

To analyze the arrangement performance in more detail, we performed simulation experiments with the following conditions.

1. All objects have the same dimensions and properties.

2. Objects have different dimensions but the same properties.

3. Objects have the same dimensions but different properties.

The objects have size of $100[\mathrm{~mm}]$ x $100[\mathrm{~mm}]$ x $150[\mathrm{~mm}]$ and mass of $1[\mathrm{Kg}]$, while the container is a box of size $0.32[\mathrm{~m}]$ x $0.27[\mathrm{~m}]$ x $0.36[\mathrm{~m}]$.

Figure $\square$ shows the resulting arrangement of condition 1 . As all the objects share the same properties, the proposed method outputs a similar arrangement for all cases. The objects on top have their bottom surfaces completely supported by the top surfaces of those below. This provides a stable packing inside the container. It is worth mentioning that there are some objects on top, e.g., orange in b), which does not contact with the object below in these simulations but, due to the proximity and proper alignment, we believe that this is not a problem for the actual robot, as it will end up on top. This result is caused by the number of particles used in PSO, which, if increased, would achieve the contacting pose for all objects, at the cost of more time.

Figure $\nabla$ shows the resulting arrangement of condition 2, i.e., objects have different dimensions but the same properties. This is a case in which all objects 
can be stacked on top of each other but the variety of dimensions create a challenge for packing. Most objects have their bottom surface well supported, even when turned to one side. In some cases, e.g., purple in a), one can expect that gravity would make the item inclined until reaching stability, which we consider an acceptable solution with room for improvement.

Figure 9 shows the resulting arrangement of condition 3, i.e., objects have the same dimensions but different properties. The proposed method packs the objects according to the rules, i.e., the rigid objects (red and orange) are placed at the bottom, the deformable objects (dark-blue and green) and deformable/breakable object (purple) are placed without anything on top, and the remaining objects (others) can be on top of rigid or below deformable and breakable. This arrangement can be considered as representative of the know-how of packing.

\section{Conclusion}

In this paper, we proposed a packing robot that first arranges various types of objects in a container considering arrangement rules and executes the planned arrangement. We regard the arrangement as a 3D bin-packing problem and plan the arrangement by optimizing the objective function for arrangement. We proposed two indices: one is related to supporting areas for stable arrangement and the other is for following the arrangement rules. We generate the robot motion using inverse kinematics, common motion planning, and the heuristics in packing that objects are stacked from the bottom. Since the space above the target position is free from collisions, the strategy to approach from the top reduces the possibility of collisions.

In the experiments, the proposed method was able to arrange eleven objects under three rules. Moreover, the robot system successfully executed the planned arrangement except for putting a tissue box. Through simulation experiments, we analyzed the arrangement performance in detail with three more conditions and discussed the results.

\section{References}

1. Amazon: Amazon Robotics Challenge (2016), https://www .amazonrobotics.com/ \#/roboticschallenge/rules

2. Causo, A., Chong, Z.H., Luxman, R., Kok, Y.Y., Yi, Z., Pang, W.C., Meixuan, R., Teoh, Y.S., Jing, W., Tju, H.S., et al.: A robust robot design for item picking. In: 2018 IEEE International Conference on Robotics and Automation (ICRA). pp. 7421-7426. IEEE (2018)

3. Chitta, S., Sucan, I., Cousins, S.: Moveit! [ros topics]. IEEE Robotics Automation Magazine 19(1), 18-19 (2012)

4. Correll, N., Bekris, K.E., Berenson, D., Brock, O., Causo, A., Hauser, K., Okada, K., Rodriguez, A., Romano, J.M., Wurman, P.R.: Analysis and observations from the first amazon picking challenge. IEEE Transactions on Automation Science and Engineering 15(1), 172-188 (2018)

5. Garcia Ricardez, G.A., El Hafi, L., von Drigalski, F.: Standing on giant's shoulders: Newcomer's experience from the Amazon Robotics Challenge 2017. In: Robotic Item Picking - Applications in Warehouse \& E-Commerce. Springer (2019 (in press)) 
6. Garcia Ricardez, G.A., Okada, S., Koganti, N., Yasuda, A., Uriguen Eljuri, P.M., Sano, T., Yang, P.C., El Hafi, L., Yamamoto, M., Takamatsu, J., Ogasawara, T.: Restock and straightening system for retail automation using compliant and mobile manipulation. Advanced Robotics 34(3-4), 235-249 (2020). https://doi.org/10.1080/01691864.2019.1698460

7. Garrett, C.R., Lozano-Perez, T., Kaelbling, L.P.: Ffrob: Leveraging symbolic planning for efficient task and motion planning. The International Journal of Robotics Research 37(1), 104-136 (2018)

8. Iwasawa, H., Hu, Y., Hashimoto, H., Imahori, S., Yagiura, M.: A heuristic algorithm for the container loading problem with complex loading constraints. Journal of Advanced Mechanical Design, Systems, and Manufacturing 10(3), JAMDSM0041 (2016)

9. Kavraki, L.E., Švestka, P., Latombe, J.C., Overmars, M.H.: Probabilistic roadmaps for path planning in high-dimensional configuration spaces. IEEE Transactions on Robotics and Automation 12(4), 566-580 (1996)

10. Kennedy, J., Eberhart, R.: Particle swarm optimization. In: IEEE International Conference on Neural Networks. pp. 1942-1948 (1995)

11. King, J.E., Cognetti, M., Srinivasa, S.S.: Rearrangement planning using objectcentric and robot-centric action spaces. In: 2016 IEEE International Conference on Robotics and Automation (ICRA). pp. 3940-3947. IEEE (2016)

12. Kuffner, J., LaValle, S.M.: Rrt-connect: An efficient approach to single-query path planning. In: IEEE Intl. Conf. on Robotics and Automation. pp. 995âĂŞ-1001 (2000)

13. Li, T.S., Liu, C., Kuo, P., Fang, N., Li, C., Cheng, C., Hsieh, C., Wu, L., Liang, J., Chen, C.: A three-dimensional adaptive pso-based packing algorithm for an iot-based automated e-fulfillment packaging system. IEEE Access 5, 9188-9205 (2017)

14. Mahler, J., Liang, J., Niyaz, S., Laskey, M., Doan, R., Liu, X., Aparicio, J., Goldberg, K.: Dex-net 2.0: Deep learning to plan robust grasps with synthetic point clouds and analytic grasp metrics. In: Robotics Science and Systems(RSS) (2017)

15. Mahler, J., Matl, M., Liu, X., Li, A., Gealy, D., Goldberg, K.: Dex-net 3.0: Computing robust robot suction grasp targets using a new analytic model and deep learning. In: IEEE International Conference on Robotics and Automation (ICRA). pp. 5620-5627 (2018)

16. Makhal, A., Goins, A.K.: Reuleaux: Robot base placement by reachability analysis. In: IEEE International Conference on Robotic Computing (IRC). pp. 137-142 (2018)

17. Martello, S., Pisinger, D., Vigo, D.: The three-dimensional bin packing problem. Operations Research 48(2), 256-267 (2000)

18. Marvel, J.A., Saidi, K., Eastman, R., Hong, T., Cheok, G., Messina, E.: Technology readiness levels for randomized bin picking. In: Proceedings of the Workshop on Performance Metrics for Intelligent Systems. pp. 109-113. PerMIS '12, ACM, New York, NY, USA (2012). https://doi.org/10.6028/NIST.IR.7876

19. Morrison, D., Tow, A.W., McTaggart, M., Smith, R., Kelly-Boxall, N., WadeMcCue, S., Erskine, J., Grinover, R., Gurman, A., Hunn, T., Lee, D., Milan, A., Pham, T., Rallos, G., Razjigaev, A., Rowntree, T., Vijay, K., Zhuang, Z., Lehnert, C., Reid, I., Corke, P., Leitner, J.: Cartman: The low-cost cartesian manipulator that won the amazon robotics challenge. In: 2018 IEEE International Conference on Robotics and Automation (ICRA). pp. 7757-7764 (2018) 
20. Nebel, B., Dornhege, C., Hertle, A.: How much does a household robot need to know in order to tidy up. In: Proceedings of the AAAI Workshop on Intelligent Robotic Systems (2013)

21. Tenorth, M., Beetz, M.: Knowrob: A knowledge processing infrastructure for cognition-enabled robots. The International Journal of Robotics Research 32(5), 566-590 (2013)

22. Zhao, X., Bennell, J.A., BektaÅ§, T., Dowsland, K.: A comparative review of 3d container loading algorithms. International Transactions in Operational Research 23(1âĂŞ2), 287-320 (2016) 


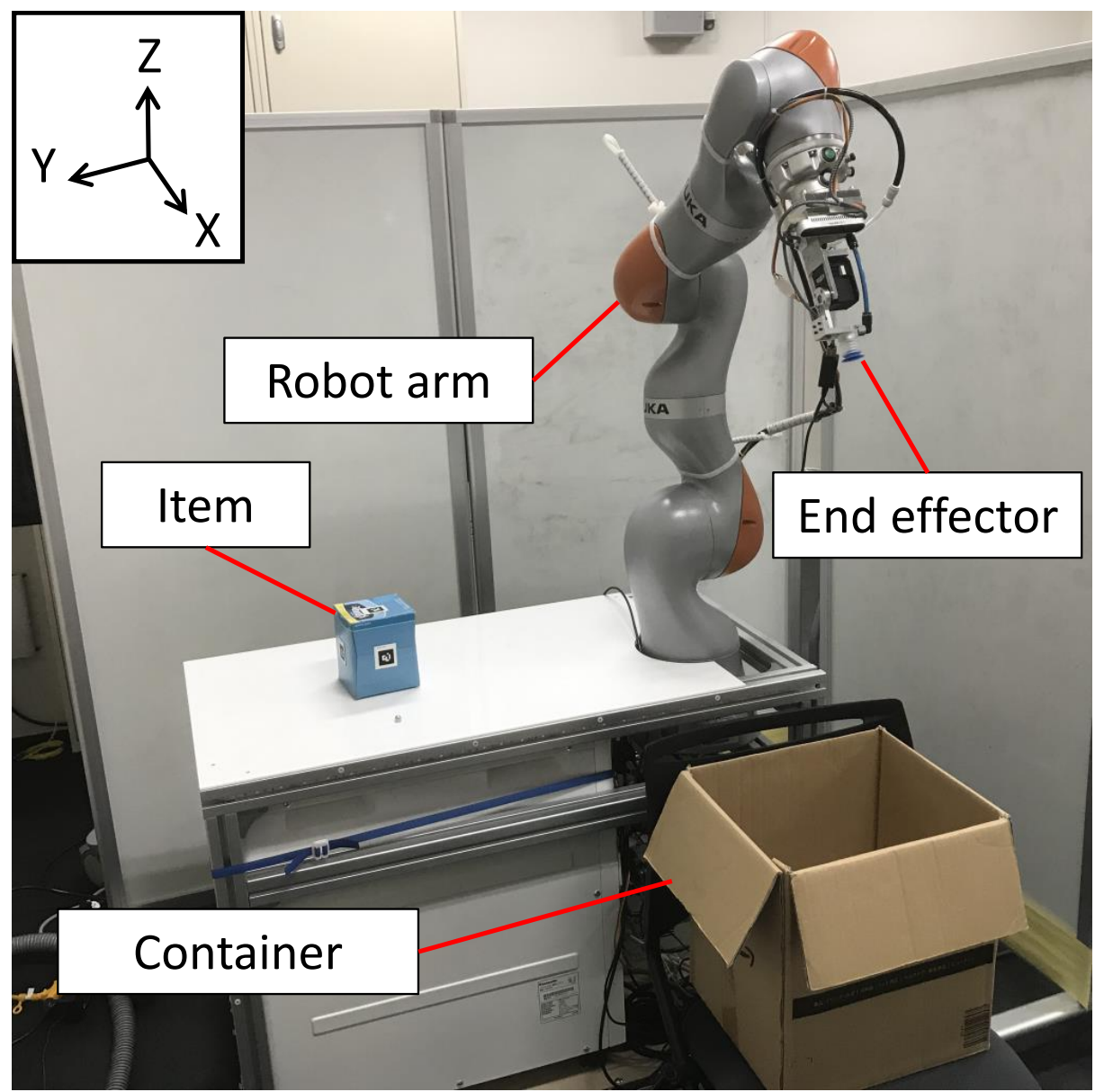

Fig. 1: Bin-packing robot system. The system plans arrangement as reducing the risk of objects getting damaged during transportation. And the system executes packing motions to place objects inside a box. 


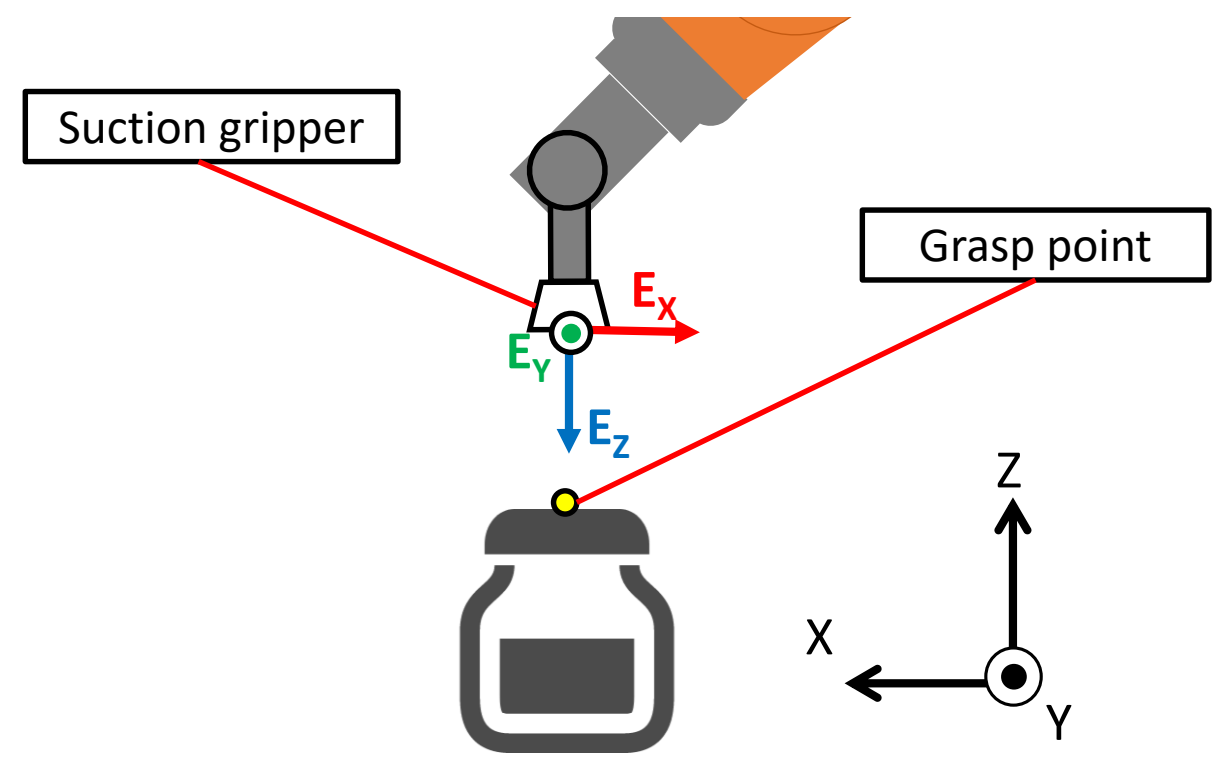

Fig. 2: The coordinate system of the suction gripper 


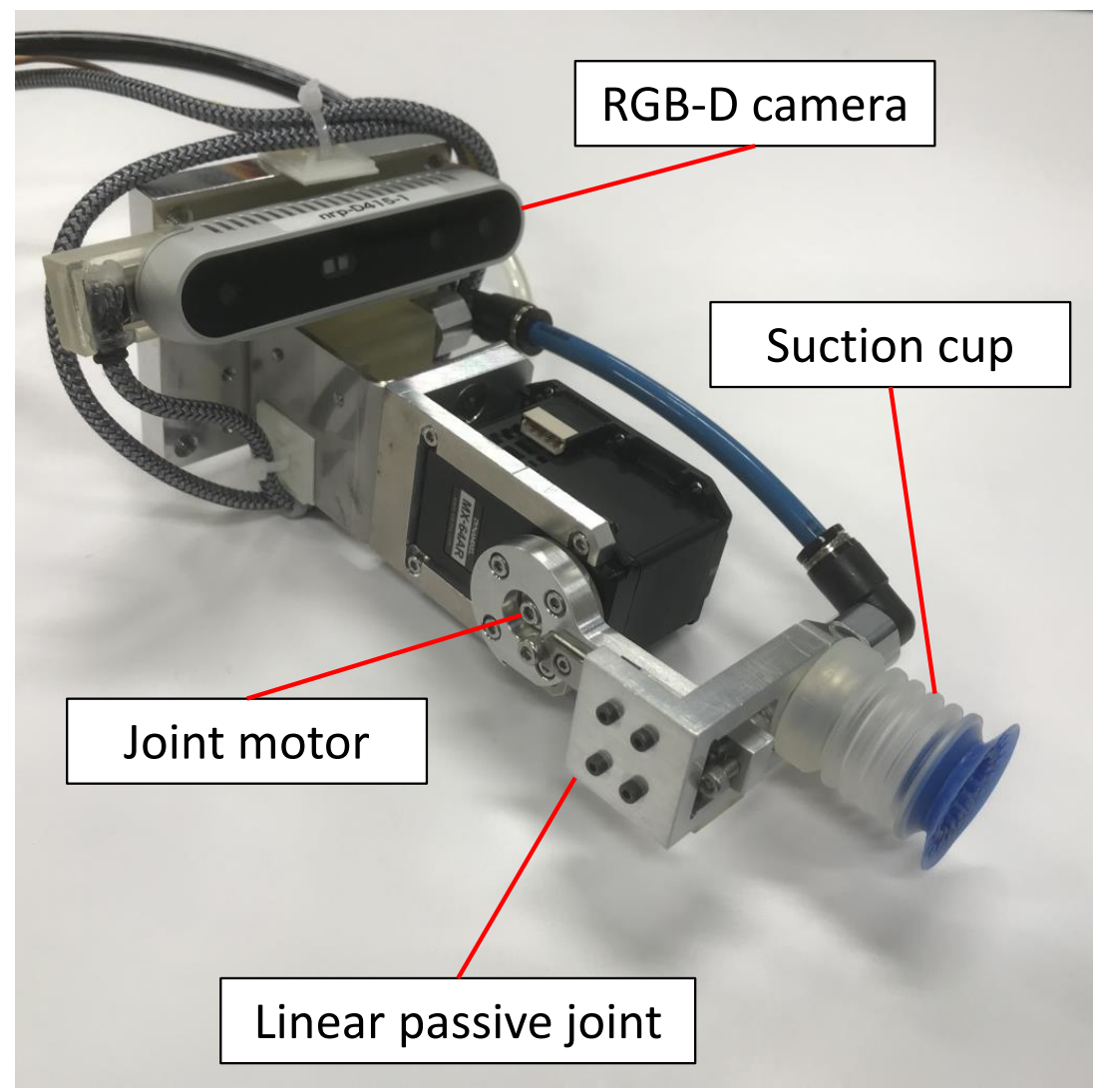

Fig. 3: End effector 
Table 6: Other items

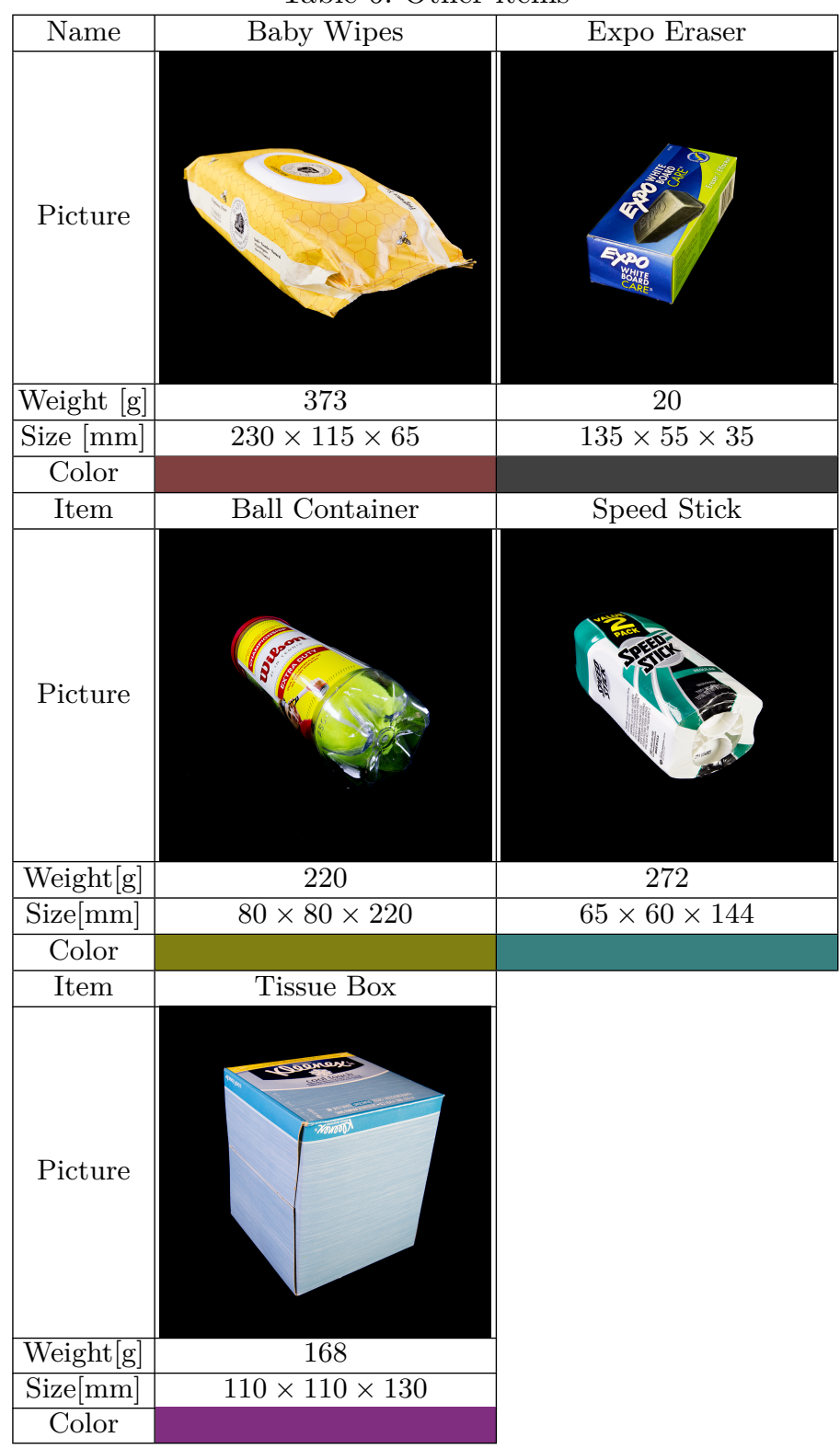




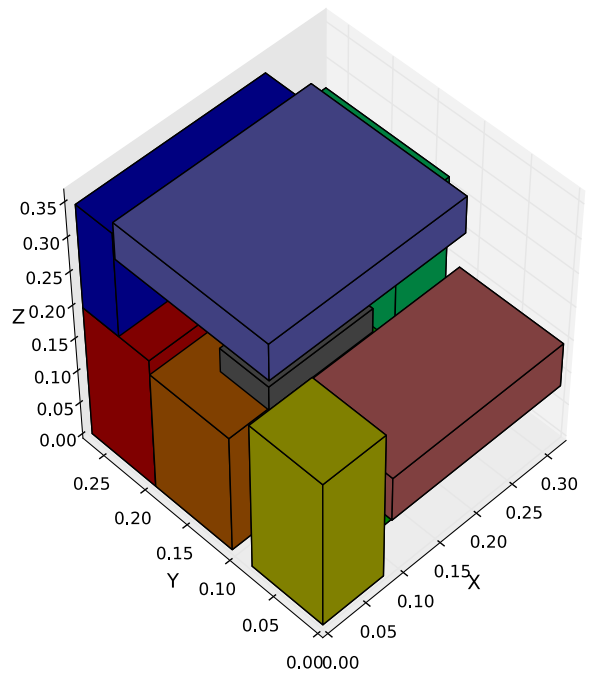

(a)

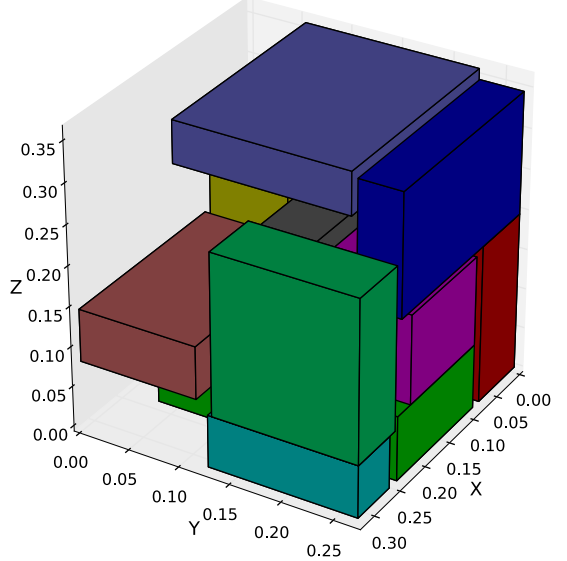

(b)

Fig. 4: Arrangement by the compared method

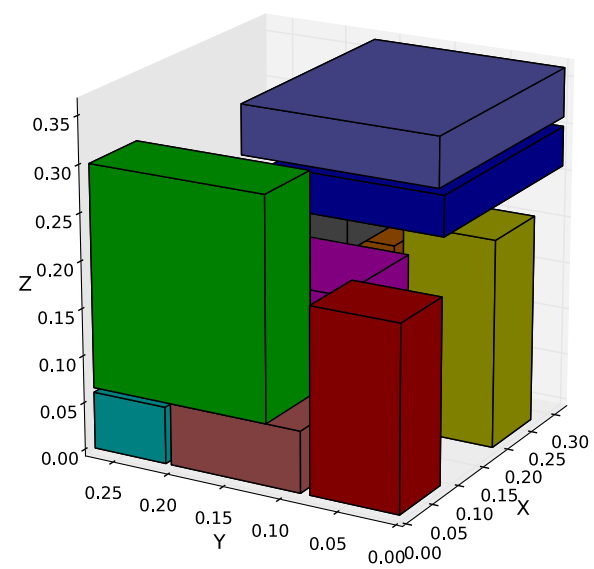

(a)

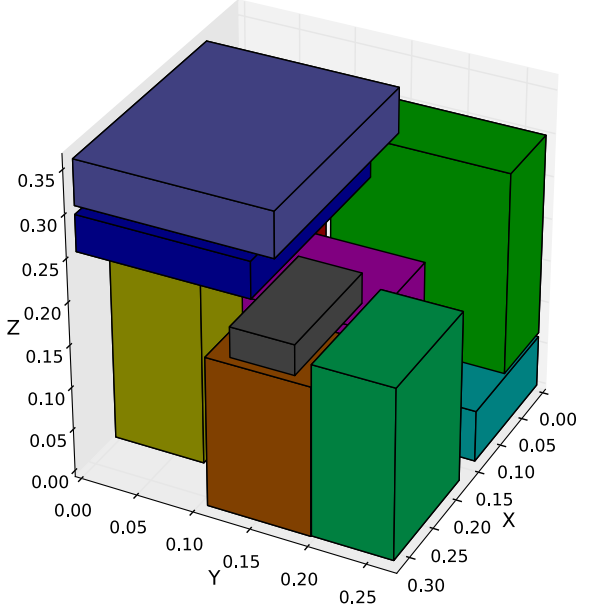

(b)

Fig. 5: Arrangement by the proposed method

Table 7: Comparison of arrangement

\begin{tabular}{|c||c|c|}
\hline Method & $\begin{array}{c}\text { Unsupported area } \\
\text { of objects }\left[\mathrm{m}^{2}\right]\end{array}$ & $\begin{array}{c}\text { Number of } \\
\text { violated rules }\end{array}$ \\
\hline Compared & 0.0646 & 2 \\
\hline Proposed & 0.0438 & 0 \\
\hline
\end{tabular}


Table 8: Result of experiment loading motion generation

\begin{tabular}{|c|c|c|}
\hline Order & Item name & Remarks \\
\hline 1 & Speed Stick & \\
\hline 2 & Baby Wipes & \\
\hline 3 & Powder pouch & \\
\hline 4 & Canned food & $\begin{array}{l}\text { Changes the position after releas- } \\
\text { ing }\end{array}$ \\
\hline 5 & Ball Container & \\
\hline 6 & Liquid detergent & Collides with the container softly \\
\hline 7 & Coffee bottle & \\
\hline 8 & Tissue Box & $\begin{array}{l}\text { Collides with another object } \\
\text { harshly }\end{array}$ \\
\hline 9 & Expo Eraser & \\
\hline 10 & Snack A & Drops softly \\
\hline 11 & Snack B & Drops softly \\
\hline
\end{tabular}
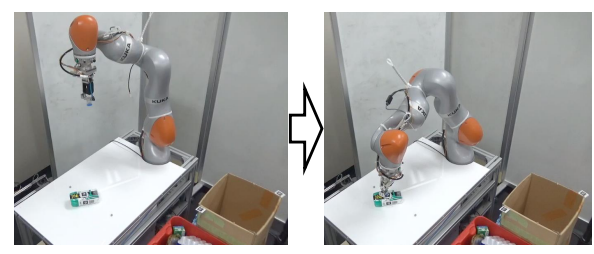

(a) Scene to pack the speed stick
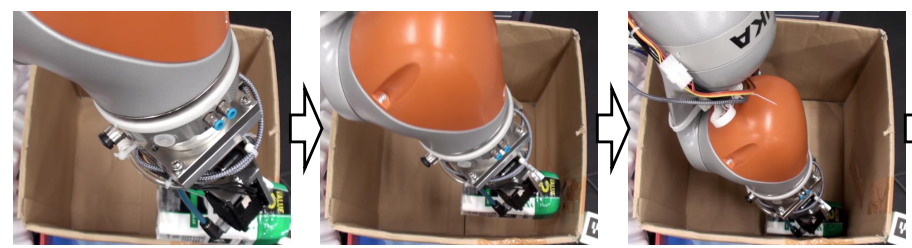

(b) View from the above of the container
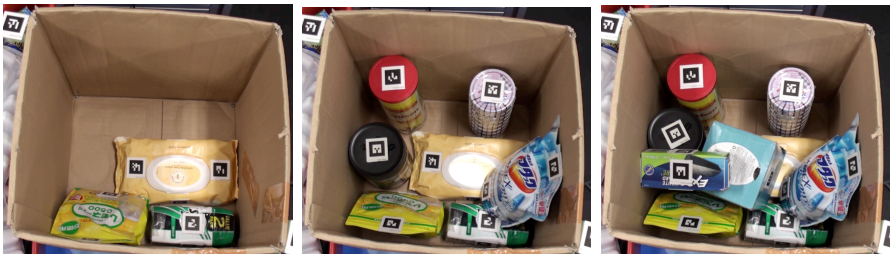

(c) Process of packing

Fig. 6: Result of robot execution
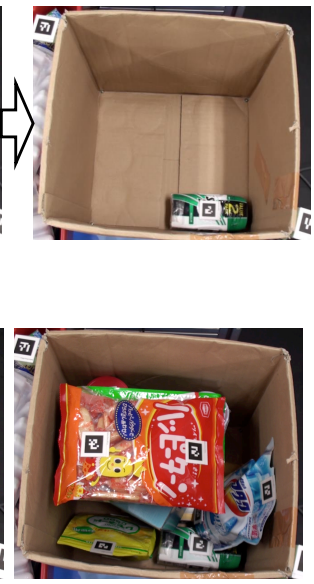

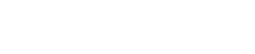




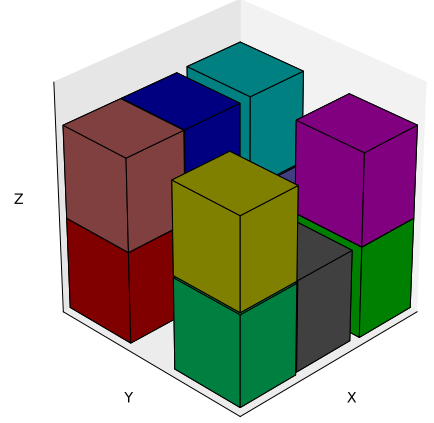

(a)

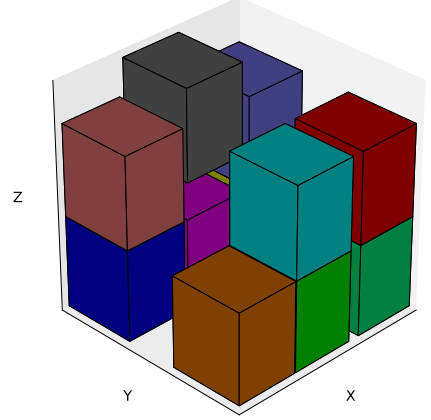

(c)

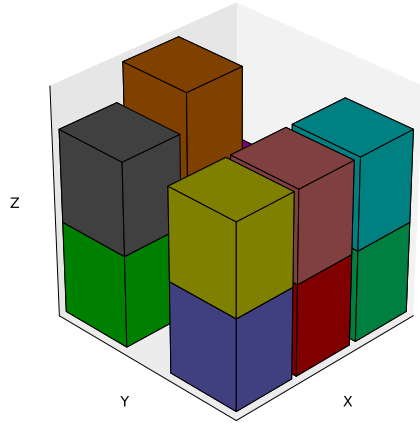

(b)

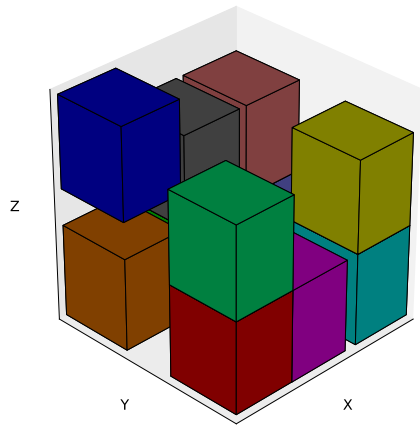

(d)

Fig. 7: Resulting arrangements with the proposed method for the case where all objects have the same dimensions and properties: a) rigid, b) deformable, c) breakable, and d) others. 

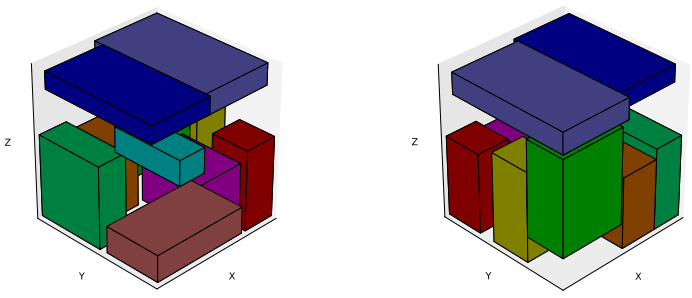

(a)
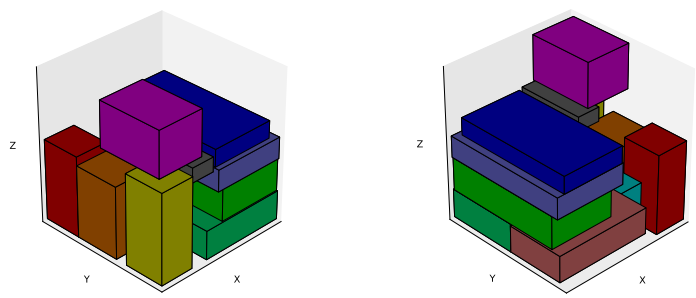

(b)
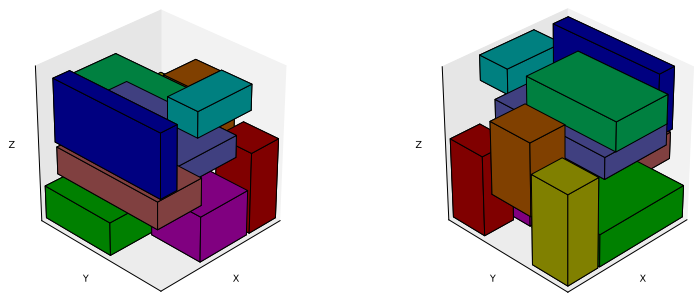

(c)
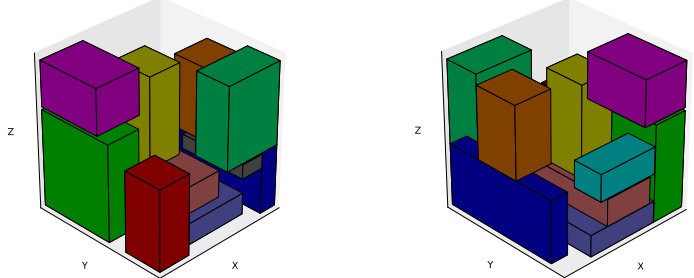

(d)

Fig. 8: Resulting arrangement with the proposed method for the case where all objects have different dimensions but similar properties: a) rigid, b) deformable, c) breakable, and d) others. Left and right columns are different views (front and back). 

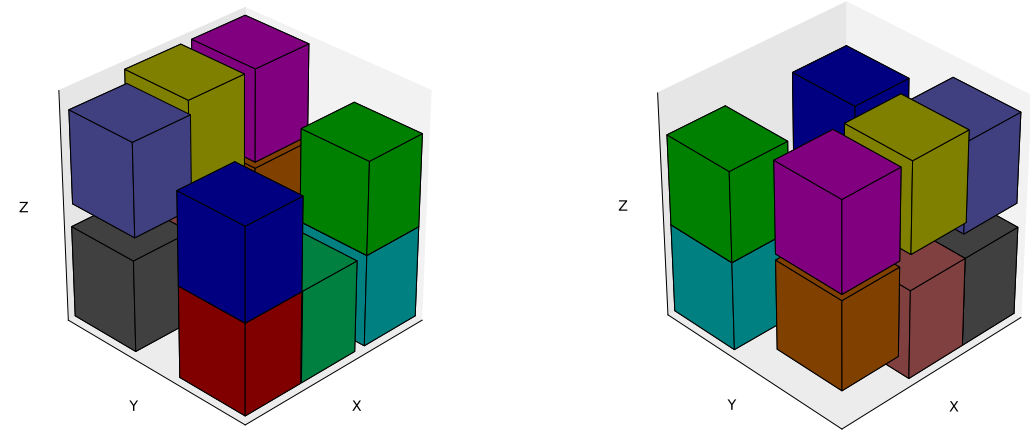

Fig. 9: Resulting arrangement with the proposed method for the case where all objects have the same dimensions but different properties. The proposed method packs the objects according to the rules, i.e., the rigid objects (red and orange) are placed at the bottom, the deformable objects (dark-blue and green) and deformable/breakable object (purple) are placed without anything on top, and the remaining objects (others) can be on top of rigid or below deformable and breakable. Left and right are different views (front and back) 